\title{
How does Social Media Affect the Psychological Well-Being of High School Students Attending Jose Marti MAST 6-12 Academy?
}

\author{
Mizael Prieto ${ }^{1}$ and Marika De Los Reyes ${ }^{\#}$ \\ ${ }^{1}$ Jose Marti MAST 6-12 Academy, Hialeah, FL, USA \\ \#Advisor
}

$\underline{\text { ABSTRACT }}$

Social media is arguably the most influential force the $21^{\text {st }}$ century has to offer more than the economy, politics, or anything in between; social media has greatly influenced everyone more than once. With a network of interconnected people so large over multiple platforms the force social media exerts over people has never been greater than now; social media has become the place where people share their opinions with the world for others to read, see, and hear. One example of its influence is the public perception on Covid-19, it increased the general fear of it and also decreased mental health among several users of social media; panic mostly spread among younger users, who expressed their concerns over social media (Ahmad \& Murad, 2020). While social media applications have a positive influence, they also have a negative influence on their users, especially teenagers. A study conducted by Dr. Ghulam Shabir, Chairman of the Department of Media Studies at The Islamia University of Bahawalpur, found that out of 279 participants, 139 agreed and 85 strongly agreed that social media has a bad influence on the participants; however, they also found that 110 of the agreed and 63 strongly agreed that social media positively influenced them (2014). With many children trying to find who they are, this study stands to find whether social media has a positive or negative effect over the psychological well-being of students in Jose Marti Mast 6-12 Academy.

\section{Literature review}

\section{Social Media}

Social media has connected us more than ever and become a place to share ideas, plans, events, and so much more across an array of different platforms and systems. While Facebook would innovate on social media; Instagram would create a platform made mainly of visual entertainment; Snapchat would create private a new way to direct message someone using pictures; and Twitter would find that a couple words can truly influence people. However, before these modern age social media giants would appear on the scene, the formation of the internet would have to occur; for more information on specific social media visit Appendix A, which contains names, release data, and other information on social media applications. In 1991 Tim Berners would create the internet, full of different ways to connect with other, blogging sites and weblogs would be the first form of social media on the internet; it would take the turn of the next century for the modern wave of social media to hit, and with that came massive change in the way people communicate (Van Dijck, 2013). This shift communication would cause change in the way we speak and write, for example abbreviations like "LMAO" and "LOL" would allow people to type faster and more effectively, of course this all came after years of social medias popularity. The popularity of certain applications has certainly dropped and risen with time and scandals. In a study done by Monica Anderson an Associate Director of Research at Pew Research Center, a nonpartisan research center, found that the social media platforms most used by teens ages 13-17 were 
YouTube (85\%) of teens using it, Instagram (72\%), Snapchat (69\%), and Facebook (51\%) (Anderson \& Jiang, 2018). This shows that social media has advanced since its beginning in the early 2000s like Van Dijck had stated before from platforms that allow people to chat to whole pages of communities. To any average teenager the amount of time they spend on any given website or application is important, but it has only been in recent years that people have brought the ability to track device usage.

A common feature on most modern devices allows people to track how long they spend time on certain applications, this allows for a more accurate read on how long a person spend on social media and on applications in general. A study, conducted by John Rooksby a researcher with a PhD in Computer Science from Manchester University, found that an application that tracks screen time on individual or multiple devices had many uses such as cutting down screen time, managing screen time, tracking activities on devices, and understanding device use (Sumter et al., 2009). Applications like this have become very useful in studies that attempt to track the amount of time students spend on their devices; for example, Anderson's study would have most defiantly increased in accuracy with the usage of a screen time tracking application. In this world of technology and modernization, the amount of time we spend on social media is equal to our unprecedented capacity to others to connect people.

When a person uses social media, it affects more the just them; the main point of social media is the connectivity of people, so whenever a person does anything on social media it affects them as well as their friends and family. At the same time, students that uses social media might be heavily affected by others in real life and online. A study found that many teen-parent relationships effected the teens relationship to the internet; a weak teen-parent relationship results in an over-reliance on internet and peers on social media, while a strong relationship with parents results in use of social media to solely entertain (Vasanth \& Swamy, 2013). Of course, relations with friends also affects the use of social media, numerous students count on their online friends as much as their real friends, so relationships with real life friends affect relationships with online friends and vice versa. An over reliance on online friends can also result in cyberbullying as the bonds of peers on the internet change due to new situations. In a study presented by Whittaker and Kowalski in the Journal of School Violence, a peer reviewed scientific journal that specifically focuses on violence and why it occurs in schools, found that 224 participants took a survey and reported that $18.2 \%$ were being cyberbullied in the last year and 55\% had at least seen cyberbullying taking place in the last year; in $50 \%$ of the cyber bulling cases the perpetrator was a friend (Whittaker \& Kowalski, 2015). The overwhelming majority of cases involving over dependence on social media or cyber bulling can cause things like poor psychological well-being; however, social media is also a place of love and support, proven by a variety very different and unique communities on social media.

\section{Psychological Well-Being}

Psychological well-being is defined as a multidimensional value that attempts to encapsulate a variety of components including a 6-factor model that connects ideas such as happiness, self-esteem, and life satisfaction to capture it all in one value (Warr, 1978). Measuring psychological well-being is no easy task as it is still considered to be somewhat theoretical; however, the value provided by psychological well-being is very much worth the work because it is one of the best tools that the scientific community has. In a study published in Educational Sciences: Theory and Practice, an international academic journal that high quality articles, described that after translating several psychological wellbeing exams into Turkish, the surveys were still able to measure the six-factor model originally created reliably and consistently by Carol Yung (Akin, 2008). Its flexibility and utility in the study will be needed, this value is best suited to this study as it is the most general way to measure whether a person is in a high or poor level of mental health without having a person take a very long and complex test. A majority of the psychological well-being exams are also very straight forward and are simple for participants to take, without too many complex and convoluted questions the participant can also answer with more accuracy than other tests. An example of a good psychological well-being questionnaire is the Oxford Happiness Questionnaire (OHQ) is based on an older questionnaire the Oxford Happiness inventory (OHI), the survey is made up of 29 questions, all having 4 selections each; the test itself is compact, easy to 
understand and administer, proving itself satisfactory (Hills \& Argyle, 2002). This is only one of a variety of psychological well-being surveys that prove more than satisfactory in order to test for such a multidimensional concept that inhabits topics such as happiness, life satisfaction, and self-esteem.

\section{Social Media Applications \& Psychological Well-Being}

Social media and psychological well-being have been shown to correlate within numerous studies, what truly remains ambiguous is whether social media causes an increase or a decrease in psychological well-being. The general consensus is that certain things can cause positive effects while other can cause negative effects, for example cyber bullying can push minors to self-harm or worse. In a study that supports Whittaker and Kowalski's findings, they argued that social media can push a minor to self-harm, especially after being cyberbullied; interestingly, the study also presented support group on social media that provide help to people who need emotional support (Memon et al., 2018). Although this study supports Whittaker's view that social media causes poor psychological well-being among teenagers, it also presents the duality of research on social media as every different facet of social media can cause a variety of positives and negatives to occur. According to Gwenn Schurgin O'Keeff, a digital media expert and M.D. pediatrician, the benefits of social media include community engagement, creative learning, growth of ideas, and connectivity to others (O'Keeffe \& Clarke-Pearson, 2011). In a similar effect to psychological well-being, social media's effects on a person are multidimensional and difficult to calculate in specificity as there a variety of factors that causes a person to use social media with frequency.

Numerous studies present a different take on social media's effect on psychological well-being, various studies assert that social media usage is merely a cause of a greater conflict in the life of a person that causes psychological distress. A study published in the Psychology of Addictive Behaviors, a journal which is part of the American Psychological Association, asserts that people not in a relationship use social media more than those that are in a relationship, mostly because of time constraints, most couples prefer to spend time together then post on social media (Andreassen et al., 2016). Moreover, this effect can also be felt with friends, colleges, parents, or anything in general; in fact, using free time to do something other social media is commonly found to help others. Most adolescents that do on screen activities such as playing video games and using social media have a lower phycological well-being, then people that do less on-screen actives like socializing in person and exercising (Twenge et al., 2018). Social media is almost used as a coping mechanism for people in psychological distress, while this dependence social media might be positive for a person this dependence might grow into a powerful addiction. Bueal Keles a nurse who mostly focuses on psychiatric patients, and a graduate from King's College London found that teenagers with an addiction to social media lack mental health and are easy targets for people that want to cyberbully others online (Keles, 2020). Social media's effect on youth are very powerful and its unprecedented effect is what causes many people to become addicted to it, whether it is a positive or negative effect. Of course, another result of the study is that social media is one of onehundred things that effect a person's psychological well-being every day, meaning social media might only play a small part in someone's psychological well-being. In a study done by Amy Orben a college research fellow at the University of Cambridge, who is focused on the connection between social media and well-being, using data from Ireland, the United States, and the United Kingdom, she found that there is little negative association between wellbeing and a person's active screen time (Orben \& Przybylski, 2019). While social media definitely influences a person, its influence might me so minuscule it equates to how much sunlight someone gets on a cloudy day; unless something life changing happens to occur over social media it is most probable that social media is not likely to significantly affect the real world that social media attempts to encompass.

\section{Current Study}

Studies on psychological well-being and social media have a variety of results; however, based on the research discussed above, I hypothesize that as social media use increases a student's psychological well-being will decreases; 
however, a student will see an increase in their psychological well-being if they use social media to a moderate degree. A baseline for average time on social media will be established during the study, this will allow us to see what healthy amount of screen time is.

This study might also be affected by the current Covid-19 epidemic; a study from Amy Orben suggests that because of the increased amount of isolation that adolescence have felt during the worldwide quarantine, social media has become a platform where they can have easy peer interaction, which would increase their psychological wellbeing (Orben et al., 2020). This can potentially and significantly change the results of the study; however, the hypothesis continues to stand as is, because this study was done in the early months of the pandemic. With society returning back to normal slowly this study might now be invalid; furthermore, results could change due to this study being concentrated in one area.

\section{Methods}

\section{Participants}

This study is non-experimental research that will use quantitative data to find a correlation between time spent on social media and psychological well-being. The study will use convenience/purposive sampling, a type of sampling that is efficient, quick, and low cost; in most situations, participants are selected simply because they are in the right place at the right time (Acharya et al., 2013). All participants in the study will be students from Jose Marti MAST 612 Academy, and within the grades of 9th to 12th, due to the majority of the school's population being of Hispanic origin, the study might very well be affected because of cultural aspects. The study will also be affected by how long students are on social media and the activities they might conduct on social media. Participants will receive a google forms survey and be asked to complete it; most questions are tailored to the study; however, the study's psychological well-being section contains the General Well-Being Schedule or the GWS.

Procedures

All procedures completed in the study were approved by Jose Marti MAST 6-12 Academy's Institutional Review Board and followed the ethical guidelines. Students in Jose Marti MAST 6-12 Academy were asked at random to take one google forms survey that contained three separate questionaries, first the demographics section, then the social media information survey, and finally the GWS which uses a 6-point Likert scale. This method was inspired by a study that investigated how students in both a private and public school use social media, using random sampling the researchers found students to take the survey on site, and their questions would be on a 5-point Likert Scale; in the end their method would work (Lu et al., 2016). However, because of Covid-19 safety restrictions the convenience sampling would have to be done at a safe distance, the survey was sent to students using links, and the survey could be taken anytime and on any device. Participants provided their gender, age, and more, but no personal information was released to the public, keeping participant data private. The data collection process took place over fourteen days; the fourteen days included sending students links to the survey and asking students who physically attend school to take it.

\section{Measures}

\section{Social Media Questionnaire}

In this study, two questionnaires are used along with a small demographics section, one questionnaire is custom made for the study (Social Media Questionnaire), and one was premade for the explicit purpose of measuring a person's psychological well-being. The Social Media Questionnaire is placed after the demographic questions and is comprised of 12 questions; the majority of these questions focus on how long a person uses social media and how long they use specific social media applications. However, a small number of the questions focus on a student's overall experience 
on social media; two of those four inquires include cyberbullying. one is a person's opinion on their experience on social media, and a one includes questions about the participant's use of social media.

\section{General Well-Being Schedule}

The GWS is a reliable questionnaire made up of 18 questions that will generally measure well-being, but it also considers six other subjects: anxiety, depression, positive well-being, self-control, vitality, and general health (Dupuy, 1977). The questionnaire has aged quite a bit, but its creator and other exam users have assured its effectiveness and timelessness. The exam itself uses a 6-point Likert scale for the first 14 questions, and the last four questions are rating questions, with a selection of 0 to 10 ; however, they only allow a participant to select even numbers between 0 and 10. Depending on the numerical score the student taking the test is placed in several categories: Positive well-being(81110), Low Positive(76-80), Marginal(71-75), Stress Problem(56-70), Distress(41-55), Serious(26-40), and Severe(025). In order to suit the purposes of the study, several editions were made to the questionnaire. Question 12, "Have you felt downhearted and blue?" was removed from the survey to shorten the survey. However, more critical is that the question itself has not aged well as the term feeling blue is outdated, and the overall generality of the question made it useless to the study. Especially when the GWS is full of more insightful questions; for example, question 4 reads, "Have you felt so sad, discouraged, hopeless, or had so many problems that you wondered if anything was worthwhile?". To accommodate the removal of this question the questionnaire's grading scale was edited so that it was proportional to a 17-question survey: Positive well-being (76-105), Low Positive(71-75), Marginal(66-70), Stress Problem(51-65), Distress(35-50), Serious(21-34), and Severe(0-20). The last four questions have also been altered so that participants are not limited to only selecting even numbers; they can pick and choose from all integers from 0 to 10 can be selected, which gives participants a more extensive range of options and gives the study precise results.

\section{Statistical Analysis}

Data collection is complete. After collecting 51 valid responses to the google forms survey created, the data is organized into a excel spreadsheet. A study published by Springer, a reputable publisher established in 1950, functioned as a guide on what types of statical analysis should be used for the conducted study as both studies are incredibly similar; the study gave way to the correlational statistics that will soon be mentioned (K1rcaburun et al., 2019). After categorizing students into different sections based on how much time they spent on social media websites and how much time they spent on social media generally. Students were organized into three categorize based on the total amount of time they spent on social media; students ranging between more than 6 hours and 4 hours were placed into the high use section, students between 3 hours and 2 hours were placed into the medium use section, and students between 1 hour and 0 hours were placed into the low use section. Students who answered more than 6 hours were registered with the number 7; which represents 7 hours; similarly, students with less than 30 minutes were represented with 0.25 and 30 minutes were represented with 0.50 . The correlation coefficient was found, comparing every student's psychological well-being score to overall time on social media, time on individual social media applications, and different ranges of time spent on social media. Besides finding the correlation between psychological well-being and the amount of time a person spends on social media, a person's opinion of social media was also tested. This came in the form of asking students whether they had a positive of negative experience on social media and also asking if they had been cyber bullied or conducted cyber bullying. To analyze the participant's use of data, their quantitative responses was transformed into $1 \mathrm{~s}$ and $2 \mathrm{~s}$, before using statical tools. After finding the correlation coefficient, the $\mathrm{p}$ value and R-squared of every set of data were calculated to assure that every set of data was not due to random chance and was an effect of what was asked.

There was no statical significance between how long a person uses social media and their psychological wellbeing. Meaning that social media has no or a limited effect on someone's psychological well-being.

\section{Results}




\section{Psychological Well-Being Scores}

Among the 51 participants in the study, the majority scored between a range of 40 to 80 , with the highest overall category being stress problem and the lowest belonging to the Serious and Severe categories, as seen in figure 1. Thus, the data shows that most participants are at least stressed due to some external cause; for example, school, home, work, or social life, but it is most definitely not due to social media.

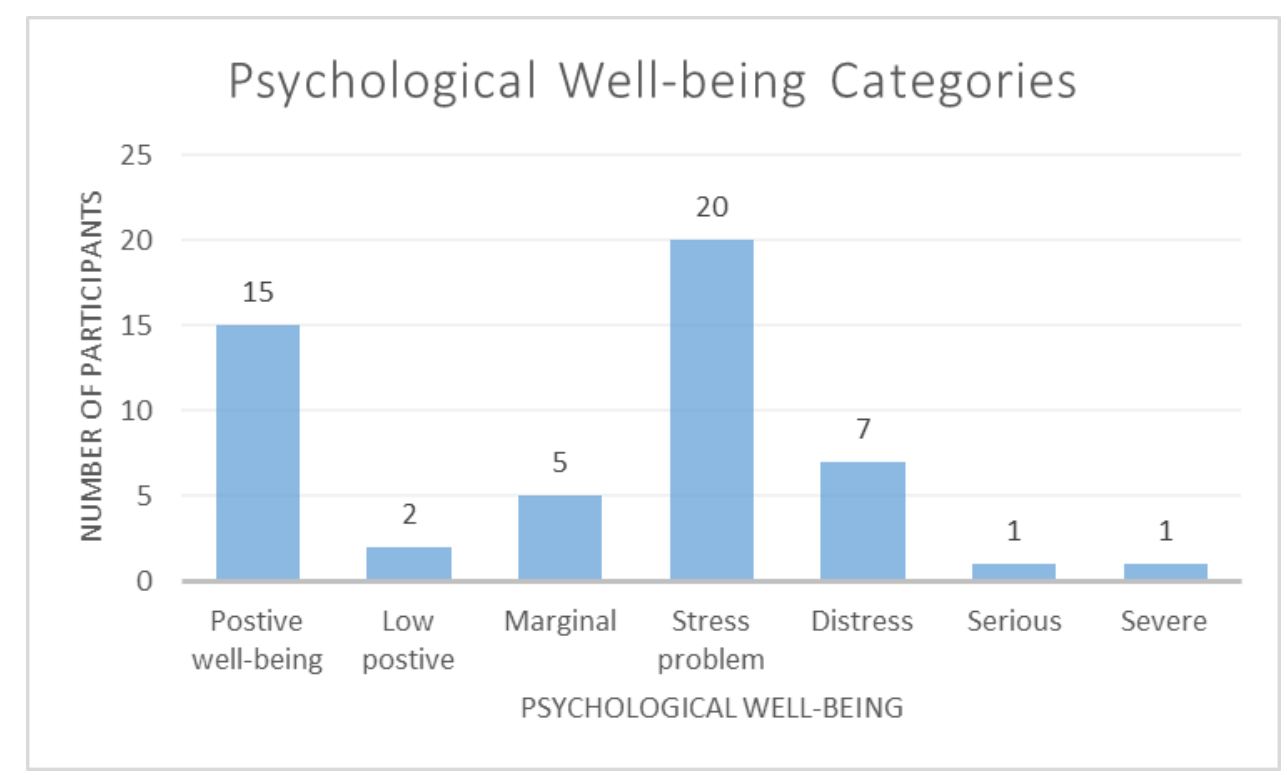

Figure 1. Number of Participants per Psychological Well-being Levels

\section{Psychological Well-being Scores and General Time Spent on Social Media}

All statistics used in the study found significantly lackluster results. The most considerable correlation belongs to the high range of time spent on social media with a correlation of 0.28032 and the one of lowest belonging to each student's overall time on social media, with a correlation of -0.004671638 . However, this extremely weak correlation is most likely due to chance as the p-value was 0.974 , which is several leagues above 0.05 , the smallest p-value necessary for a statistic to be considered statically significant. Furthermore, the R-squared value confirmed the insignificance of the correlation, with a value of 0 . Thus, both statistical tests, $\mathrm{p}$-value, and R-squared presents the possibility that the study conducted did not yield any significant or even worthy results. 


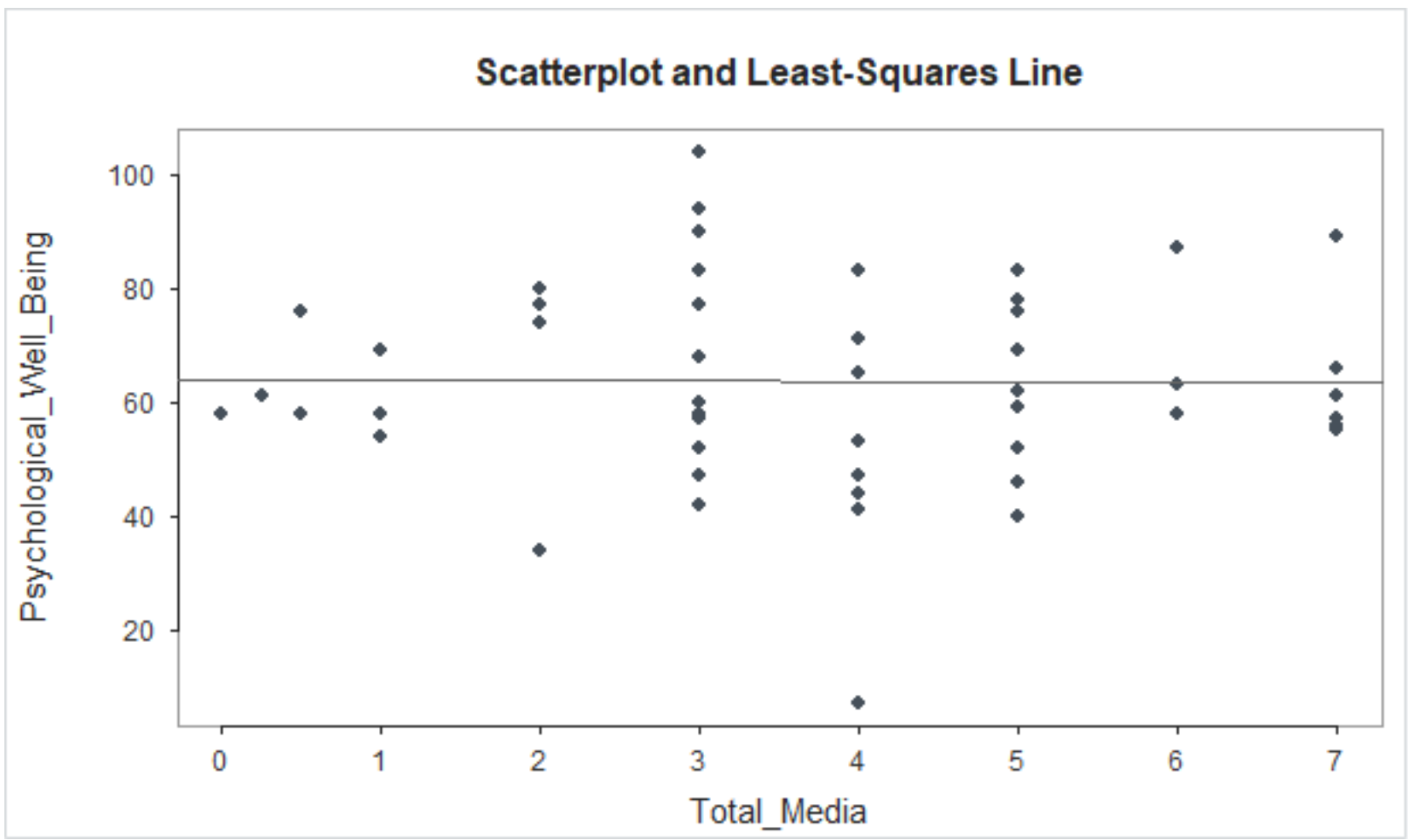

Figure 2. Relationship Between Media Usage and Psychological Well-being

\section{Psychological Well-being Scores and Time Spent on individual Social Media Platforms}

Like the total amount of time students spend on social media, time on individual platforms is also statistically insignificant, as seen in Table 1. Most individual social media platforms are unusable due to their low R-squared and high p-value; however, among the individual platforms, there is an outlier, that outlier being Tik Tok. The lowest p-value of 0.082 and the highest R-squared of 0.060 is still not considered statistically significant, but it is more valuable than any other statistics calculated through the whole study. Its more significant correlational value of 0.245673431 also makes it useful; even though the statistic itself is not significant, there is still a lot to be said about Tik Tok.

Table 1. Statistical Results of All Individual Social Media Platforms

\begin{tabular}{l|lll}
\multicolumn{1}{l}{$\begin{array}{l}\text { Social Media } \\
\text { Facebook }\end{array}$} & porrelation & Ralue & 0.002 \\
Twitter & -0.041815354 & 0.771 & 0.004 \\
Instagram & 0.192326064 & 0.644 & 0.037 \\
Tik Tok & 0.245673431 & 0.176 & 0.060 \\
YouTube & 0.136791298 & 0.082 & 0.019 \\
Reddit & -0.07912178 & 0.338 & 0.006 \\
Snapchat & -0.024491168 & 0.581 & 0.001
\end{tabular}




\section{Psychological Well-being Scores and Specific Time Spent on Social Media}

Participants were divided into three groups, considering the amount of time spent on social media; overall, this division is meant to find if correlation goes up depending on the amount of time someone spends on social media. The categories included: more than 6 hours to 4 hours, 3 hours to 2 hours, and 1 hour to 0 hours. The 6-to-4-hour group had a correlation of 0.28032 , the 3-to-2-hour group had a correlation of - 0.0133 , and the 1-to-0-hour group had a correlation of 0.06826 . The correlation of all three groups is entirely insignificant, meaning that, most likely, there is not a correlation between the specific amount of time someone spends on social media and their psychological well-being. Although the 6-to-4-hour group is the highest correlation throughout the whole study, it is useless as it shares a p-value and R-squared with the overall time each student is on social media. The p-value for each category was 0.974 , and the R-squared for each category was 0 .

\section{Psychological Well-being Scores and Participant's Use of Social Media}

Another factor tested in the survey was a participant's experience throughout their use of social media; four of these questions were added; however, only three of the questions will be examined. Two questions asked whether the participant had been cyberbullied or experienced cyberbullying, while the other question asked whether the participant's experience was positive or negative. The experience question has a correlation of - 0.098069677 , a R-squared of 0.010, and a p-value of 0.494 . The cyberbullying victim question a correlation of 0.031987939 , a R-squared of 0.001 , and a $\mathrm{p}$-value of 0.824 . The cyberbullying question has a correlation of -0.070239834 , a R-squared of 0.005 , and a p-value of 0.624. Similar to the rest of the results of this experiment, the data is insignificant due to low correlation, high pvalue, and a low R-squared. However, in an exciting turn of events, most participants responded that they had a positive experience on social media.

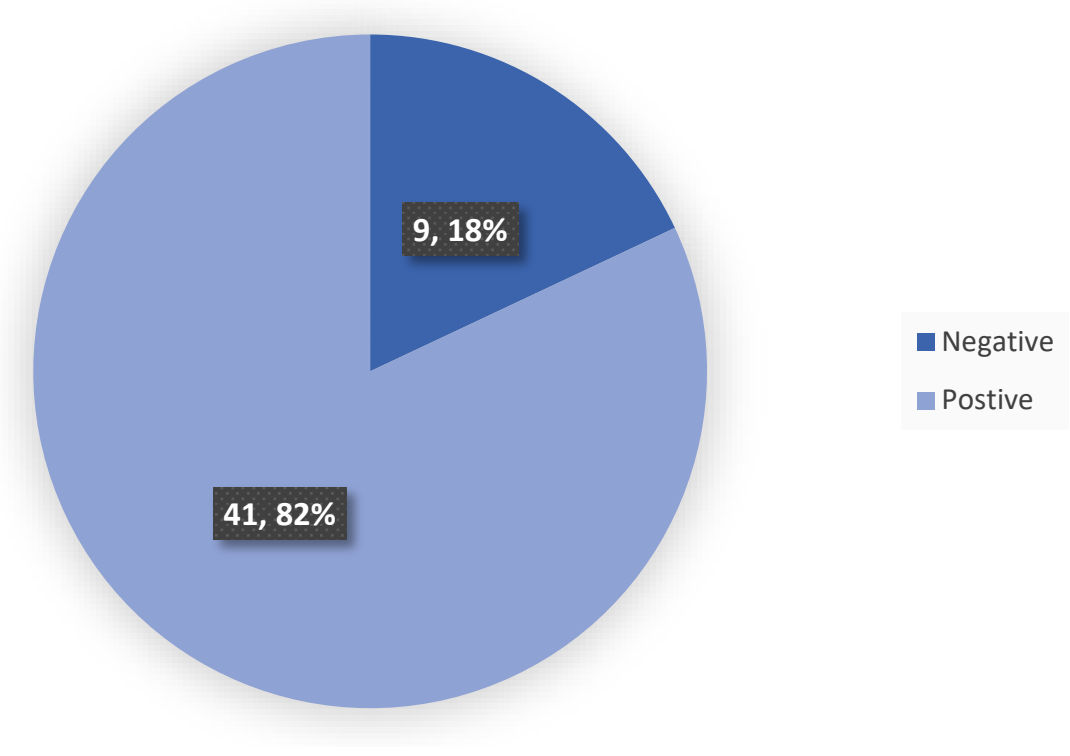

Figure 3. Comparing Participants' Experiences on Social Media

\section{Discussion}

After analyzing the results of this study, it is clear that the study proved to yield no practical or useable results. Visible in Figure 1, there is a weak correlation and an even weaker R-squared and p-value between the participant's psychological well-being and their overall time spent on social media, and these results set the trend for the whole study. 
Furthermore, every statical test conducted on every set of data came back with lackluster results, which nullifies the studies original hypothesis that significant use of social media will negatively affect a high school student attending Jose Marti MAST 6-12 Academy while using social media for a low or moderate amount will improve psychological well-being. Instead, this study found that social media might not be linked to psychological well-being and that psychological well-being is actually linked to various other factors.

\section{Implications}

This study does not impact the psychological well-being and social media research community, primarily due to the study's inability to prove that the results gathered are significant. However, this study proves that there is an enormous waste of energy towards stopping children from engaging in social media activities. In figure 3 , it is visible that most students derive positive emotions and experiences from social media; if anything, there should be a larger focus on what affects students in real life; as discussed earlier, family relations and relationship status can be detrital to social media usage and psychological well-being (Andreassen et al., 2016; Vasanth \& Swamy, 2013). It seems like social media acts as an escape from reality for most or at least a joyful hobby.

In future studies, psychological well-being should be compared to more specific social media variables, like a specific app, trends on apps, and social media communities; by focusing on more specific facets of social media, studies might find better results. Of course, studying a significant portion of social media will not yield results, and this might be due to the multi-faceted nature of psychological well-being and the infinite possibilities present in social media. However, by studying a specific variable of social media, it becomes possible to have at least some form of control over the madness that is the internet.

\section{Conclusion}

This study did not produce any significant results that will affect the majority of the scientific research community due to the underwhelming results formulated throughout this study. There was no connection between psychological well-being and social media usages even after testing general time spent on social media, time spent on individual social media applications, and experience on social media. This lack of correlation expresses the sentiment that social media is not correlated with psychological well-being; however, it is most likely that those results were completely due to random chance. This is because of the low R-squared and high p-value every data set had, which impresses the idea that the study was a distinct failure, but from a different angle, it is positive.

Numerous people spend their day interacting with others over social media; the whole reason this study began was because of the social media explosion sue to Covid-19; because of this constant use of social media, the world worries that humans are becoming unstable as individuals. Limited face-to-face interactions hurt us, but that does not mean that social media negatively influences the minds of young ones; if anything, it is a powerful positive source from which many draw their strength in times of need. It is the duty of the human race to pause and consider the worth of investing time into stopping something positive. While this study proves to be useless statistically, it shows that maybe it is time to deal with problems in the real world that genuinely affect people's lives instead of blaming them on social media.

\section{Limitations}

This study contained a plethora of limitations that may have significantly influenced the results of this study; after examining the limitations of this study, it is entirely possible that this study failed to yield results due to three factors a lack of respondent honesty, leaving large sections of the school out of the study, and Covid-19 restrictions. 
A participant's honesty is a large part of this study; for that reason, researchers are forced to place that trust in participants, but the issue with this trust is that it is easily betrayed. Due to the sensitive nature of this study, numerous participants might have falsified their answers to better hide their lack of psychological well-being or their overbearing use of social media. Unfortunately, hiding these answers caused a colossal infraction in the study's results as false results on a single section of the questionnaire would invalidate a participant's results. To address this issue in future studies, it would be wise to add questions in the demographics section of the questionnaire to confirm the validity of a participant's results.

Even confirming the validity of these results would still not be enough to rectify the results of this study because there is still a large part of the student population of Jose Marti MAST 6-12 Academy that was not sampled throughout the 2-week survey period. For example, of the 51 participants, zero were in the 9 th grade, and 29 were in the 11th grade, most likely due to my grade level being the same. Similarly, 42 students who took the survey attended school physically compared to 9 who attended school online. Such great deviations in participants would have made the study not apply to the whole of Jose Marti MAST 6-12 Academy, which would have rendered the study invalid to specific groups at the school. To counteract this, using stratified sampling instead of random sampling would allow a perfectly even split for all grade levels at the least. This large difference in respondents is most likely due to the difficulties that come with giving out a survey during a worldwide pandemic, like low response rates from students who take classes online.

Covid-19 itself was a significant factor in this study as it could have been a prominent cause of low psychological well-being or high social media use; the virus itself was, in large part, a cause of this study because it is what causes the social media boom at the beginning of 2020. In large part, the reason this study could not gain more participants was because of Covid-19. Without such restrictions, there would have been a 70 to $80 \%$ increase in the number of participants as 42 participants of the study sampled directly from the physical school. With more straightforward access to the other half of the school, the study could have easily flourished into a study with more than 100 participants. It would be wise to wait for this pandemic to be over before conducting studies similar to this, as Covid-19 presents a confounding variable that is difficult to measure due to its broad effect on individuals and groups. However, it also presents an interesting variable to study on its own, a future study on how Covid-19 has impacted social media use or psychological well-being would be fascinating.

\section{Acknowledgments}

I would like to thank my mother and father for supporting me in my endeavor to purse a college education and to my amazing friends, I could not have done this without you.

\section{References}

Acharya, A. S., Prakash, A., Saxena, P., \& Nigam, A. (2013). Sampling: Why and how of it. Indian Journal of Medical Specialties, 4(2), 330-333

Ahmad, A. R., \& Murad, H. R. (2020). The impact of social media on panic during the COVID-19 pandemic in Iraqi Kurdistan: online questionnaire study. Journal of Medical Internet Research, 22(5), e19556.

Akin, A. (2008). The scales of psychological well-being: a study of validity and reliability. Educational sciences: Theory and practice, $8(3), 741-750$.

Anderson, M., \& Jiang, J. (2018). Teens, social media \& technology 2018. Pew Research Center, $31,2018$. 
Andreassen, C. S., Billieux, J., Griffiths, M. D., Kuss, D. J., Demetrovics, Z., Mazzoni, E., \&amp; Pallesen, S. (2016). The relationship between addictive use of social media and video games and symptoms of psychiatric disorders: A large-scale cross-sectional study. Psychology of Addictive Behaviors, 30(2), 252262. https://doi.org/10.1037/adb0000160

Dupuy, H. J. (1977). General well-being schedule. Quality of Life Research: An International Journal of Quality of Life Aspects of Treatment, Care \& Rehabilitation.

Hills, P., \&amp; Argyle, M. (2002). The Oxford happiness Questionnaire: A compact scale for the measurement of psychological well-being. Personality and Individual Differences, 33(7), 1073-1082. https://doi.org/10.1016/s0191-8869(01)00213-6

Keles, B., McCrae, N., \& Grealish, A. (2020). A systematic review: the influence of social media on depression, anxiety and psychological distress in adolescents. International Journal of Adolescence and Youth, 25(1), $79-93$

Kırcaburun, K., Kokkinos, C. M., Demetrovics, Z., Király, O., Griffiths, M. D., \& Çolak, T. S. (2019). Problematic online behaviors among adolescents and emerging adults: Associations between cyberbullying perpetration, problematic social media use, and psychosocial factors. International Journal of Mental Health and Addiction, 17(4), 891-908.

Lu, J., Hao, Q., \& Jing, M. (2016). Consuming, sharing, and creating content: How young students use new social media in and outside school. Computers in Human Behavior, 64, 55-64.

Memon, A. M., Sharma, S. G., Mohite, S. S., \& Jain, S. (2018). The role of online social networking on deliberate self-harm and suicidality in adolescents: A systematized review of literature. Indian journal of psychiatry, $60(4), 384$.

O'Keeffe, G. S., \& Clarke-Pearson, K. (2011). The impact of social media on children, adolescents, and families. Pediatrics, 127(4), 800-804.

Orben, A., \& Przybylski, A. K. (2019). Screens, teens, and psychological well-being: evidence from three time-usediary studies. Psychological science, 30(5), 682-696.

Orben, A., Tomova, L., \& Blakemore, S. J. (2020). The effects of social deprivation on adolescent development and mental health. The Lancet Child \& Adolescent Health.

Rooksby, J., Asadzadeh, P., Rost, M., Morrison, A., \& Chalmers, M. (2016, May). Personal tracking of screen time on digital devices. In Proceedings of the 2016 CHI conference on human factors in computing systems (pp. 284-296).

Shabir, G., Hameed, Y. M. Y., Safdar, G., \& Gilani, S. M. F. S. (2014). The impact of social media on youth: A case study of bahawalpur city. Asian Journal of Social Sciences \& Humanities, 3(4), 132-151.

Sumter, S. R., Bokhorst, C. L., Steinberg, L., \& Westenberg, P. M. (2009). The developmental pattern of resistance to peer influence in adolescence: Will the teenager ever be able to resist?. Journal of adolescence, 32(4), $1009-1021$ 
Twenge, J. M., Martin, G. N., \& Campbell, W. K. (2018). Decreases in psychological well-being among American adolescents after 2012 and links to screen time during the rise of smartphone technology. Emotion, 18(6), 765-780. https://doi.org/10.1037/emo0000403

Van Dijck, J. (2013). The culture of connectivity: A critical history of social media. Oxford University Press.

Vasanth, R., \& Swamy, S. (2013, July). Social Media's Impact on Teenagers. In International Conference on CrossCultural Design (pp. 477-485). Springer, Berlin, Heidelberg.

Warr, P. (1978). A study of psychological well-being. British Journal of Psychology, 69(1), 111-121.

Whittaker, E., \& Kowalski, R. M. (2015). Cyberbullying via social media. Journal of school violence, 14(1), 11-29. 